\title{
Los nuevos barrios en construcción, en el municipio madrileño. ¿Una solución al problema de la vivienda de la capital?
}

\author{
José Miguel Santos Preciado *
}

\begin{abstract}
RESUMEN
Desde su primitiva aprobación en 1993, la operación de los PAUs constituyó la primera gran apuesta política del gobierno popular, en su intento de poner en el mercado del suelo nuevas áreas de expansión, en el interior de la ciudad central. En este sentido, se pretendia modificar el tradicional modelo territorial madrileño, de expansión residencial periférica, con el objeto de evitar el declive demográfico de la capital. La perspectiva de más de diez años de gobierno municipal permite aventurar

que una parte importante de los objetivos propuestos no se han cumplido y lo que es aún más grave, el problema de la vivienda se ha acentuado hasta límites insoportables. El crecimiento del parque inmobiliario residencial, hecho incontestable acaecido con el auge

de la construcción de los últimos
\end{abstract}

\section{ABSTRACT}

Since their original adoption in 1993, the operation of the Programmes of Town Planning Conduct (Spanish abbreviation "PAU"s) has constituted the first great political bet of the government of the Spanish Popular Party, in the attempt to place new areas of expansion in the interior of the city centre on the marketplace for land. On this basis, the aim was to alter the traditional territorial model in Madrid of residential expansion on the periphery, with the object of averting the capital's demographic decline. The perspective of more than ten years of municipal government allows one to venture that a significant part of the proposed objectives has not been fulfilled, and, more seriously, the housing problem has increased to intolerable extremes. The growth of the pool of residential property, an undeniable fact which has taken place with the construction boom

* Universidad Nacional de Educación a Distancia. 
años, ha venido acompañado del incremento desorbitado de los precios y de una progresiva disminución del número de viviendas de protección en el mercado, lo que ha alejado a las capas de población de menor nivel de renta de la posibilidad de acceso a este bien tan fundamental.

PALABRAS CLAVE: política de vivienda, mercado del suelo, desarrollo urbano. in recent years, has been accompanied by a disproportionate increase in prices, and a progressive decrease in the number of protected dwellings in the marketplace, which has distanced the strata of population that have lower incomes from the possibility of access to this fundamental asset.

KEY WORDS:

housing policy, market for land, urban development.

\section{INTRODUCCIÓN}

Uno de los problemas más graves de las principales ciudades españolas es, sin duda, la falta de viviendas sociales. Las necesidades de vivienda para determinados colectivos de población, entre los que destacan los jóvenes y gran parte de los inmigrantes extranjeros, han convertido, desde hace años, a la demanda residencial insolvente en una de las cuestiones más espinosas que los gobiernos de los diversos ámbitos territoriales de nuestro país deben acometer. La llegada del Partido Popular al gobierno municipal de la capital madrileña estuvo acompañada, desde sus inicios, por los buenos deseos de dar una respuesta adecuada al grave déficit residencial, hasta entonces, acumulado. Con esta intención, "en 1992, el Ayuntamiento de Madrid puso en marcha la operación de los Programas de Actuación Urbanística (PAUs) con el propósito de colocar en el mercado un considerable volumen de suelo urbanizado con el que hacer frente a la importante bolsa de demanda de vivienda, a precio asequible, generada en Madrid, durante los años anteriores" (Ayuntamiento de Madrid, 2002). La apuesta era arriesgada y suponía la realización de un esfuerzo titánico al pretender cambiar el tradicional modelo territorial, de expansión residencial periférica, frenando la expulsión de los madrileños hacia áreas de hábitat suburbano, con el objeto de evitar el declive demográfico de la capital. La visión panorámica de más de diez años de gobierno municipal permite aventurar que una parte importante de los objetivos propuestos no se han cumplido y lo que es aún más grave, el problema de la vivienda se ha acentuado hasta límites insoportables. El crecimiento del parque inmobiliario residencial, hecho incontestable acaecido con el auge de la construcción de los últimos años, ha venido acompañado del incremento desorbitado de los precios y de una progresiva dis- 
minución del número de viviendas de protección en el mercado, lo que ha alejado a las capas de población de menor nivel de renta de la posibilidad de acceso a la este bien tan fundamental.

\section{LA OPERACIÓN URBANISTICA DE LOS PROGRAMAS DE ACTUACIÓN URBANISTICA}

Desde su primitiva aprobación en $1993^{1}$, la operación de los PAUs constituyó la primera gran apuesta política del gobierno popular, en su intento de poner en el mercado del suelo nuevas áreas de expansión, en el interior de la ciudad central. La actuación urbanística se emprendió, en un primer momento, sobre una superficie de 2.258,8 hectáreas de superficie total, repartidas en seis programas diferentes, que pretendian la construcción de 70.205 viviendas, de las cuales el $66 \%$ estarían sometidas a algún régimen de protección y el resto serían libres ${ }^{2}$ (Nasarre, F. y RodríguezAvial, L., 1995). En el momento presente, el número de viviendas a construir se ha incrementado ligeramente, habiendo disminuido el porcentaje de las que participan de algún tipo de protección (el 55.5\% del total) (tabla 1). La importancia de la operación emprendida puede deducirse del número de potenciales habitantes que correspondería al desarrollo de las nueva área urbanas, que alcanzaría la cifra de 223.000 , lo que representaría el $7.6 \%$ del total de la población de la ciudad de Madrid el año $2001^{3}$.

Tabla 1. Distribución de viviendas a construir en los PAUS de Madrid capital

\begin{tabular}{lrrrrr}
\hline Nuevos barrios & Libre & Protegida & VPO & VPT & \multicolumn{1}{c}{ Total } \\
\hline Montecarmelo & 3.882 & 4.655 & 2.850 & 1.815 & 8.537 \\
Las Tablas & 5.021 & 7.251 & 3.450 & 3.801 & 12.272 \\
Sanchinarro & 5.622 & 7.946 & 3.837 & 4.109 & 13.568 \\
Arroyo del Fresno & 1.498 & 1.494 & 760 & 827 & 2.992 \\
Vallecas & 12.240 & 13.806 & 7.214 & 6.592 & 26.046 \\
Carabanchel & 5.224 & 6.126 & 3.386 & 2.740 & 11.350 \\
\hline \multicolumn{1}{c}{ Total } & 33.245 & 37.451 & 21.404 & 19.884 & 74.537 \\
\hline
\end{tabular}

año

La aprobación inicial fue el 26 de febrero de 1993 y la provisional el 28 de julio del mismo 2

Las actuaciones protegidas estaban dirigidas a aquellas personas con ingresos comprendidos entre 2.5 y 7.6 veces el salario interprofesional.

3 Datos extraídos de los resultados provisionales del censo del 2001 , ofrecidos por el Instituto de Estadistica de la CAM. 
La distribución espacial de los barrios de nueva construcción puede apreciarse en el plano de la fig. 1. De las seis nuevas zonas residenciales, cuatro se localizan en la periferia norte de la ciudad, las de Arroyo del Fresno, Montecarmelo, Las Tablas y Sanchinarro, otra lo hace en el este, el Ensanche Villa de Vallecas, y, finalmente, la de Carabanchel en la periferia sur.

De los PAUs a desarrollar en el sector norte de la ciudad (fig. 2), el de Sanchinarro, situado en el distrito de Hortaleza, en el barrio de Valdelasfuentes, sobre un espacio triangular que supera las 400 has, limitado por la autovía de Burgos y la $M-40$, es el que se encuentra en el estadio más avanzado de la promoción. A noviembre de 2002, un total de 6.789 viviendas disponían ya de licencia de construcción ${ }^{4}$. Los otros, Las Tablas, Montecarmelo y Arroyo del Fresno se hallan localizados sobre el distrito de Fuencarral, lindando con el monte del Pardo, lo que puede suponer un

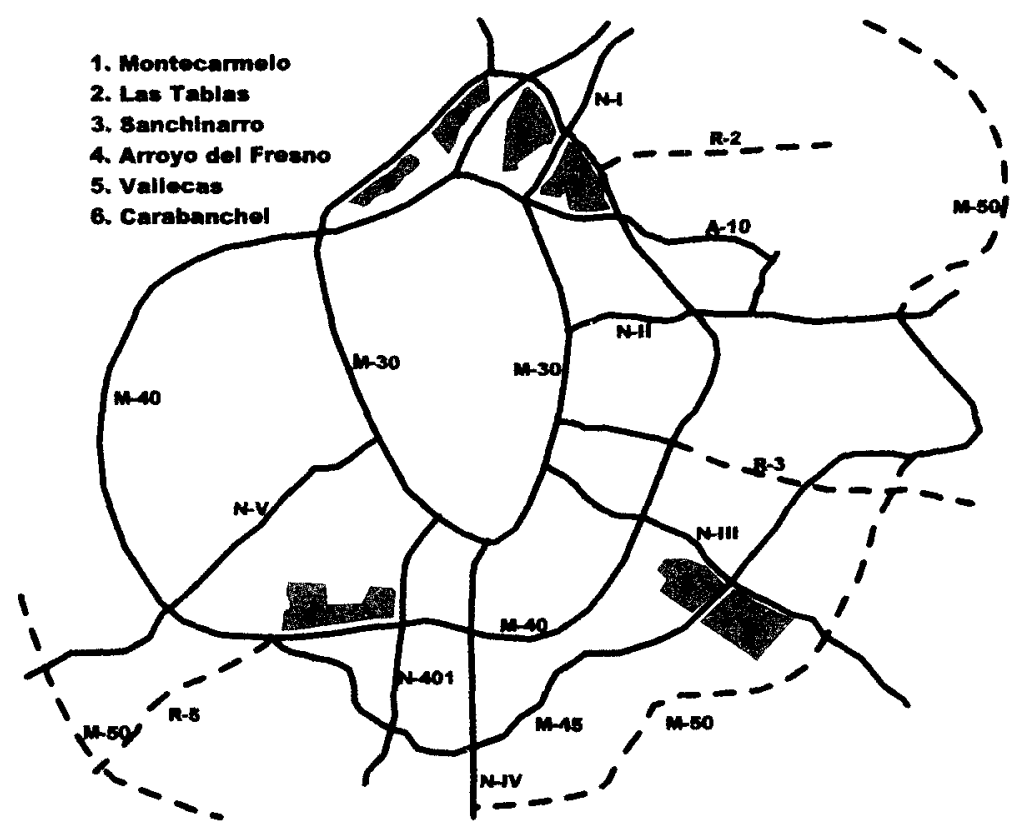

Fig. 1. Localización de los nuevos barrios de construcción (PAUS) de Madrid. Fuente: Elaboración Personal (EP).

Según datos ofrecidos por la Asociación de Promotores Inmobiliarios (Asprima) y publicados el 20-12-2002, en el periódico "El País". 


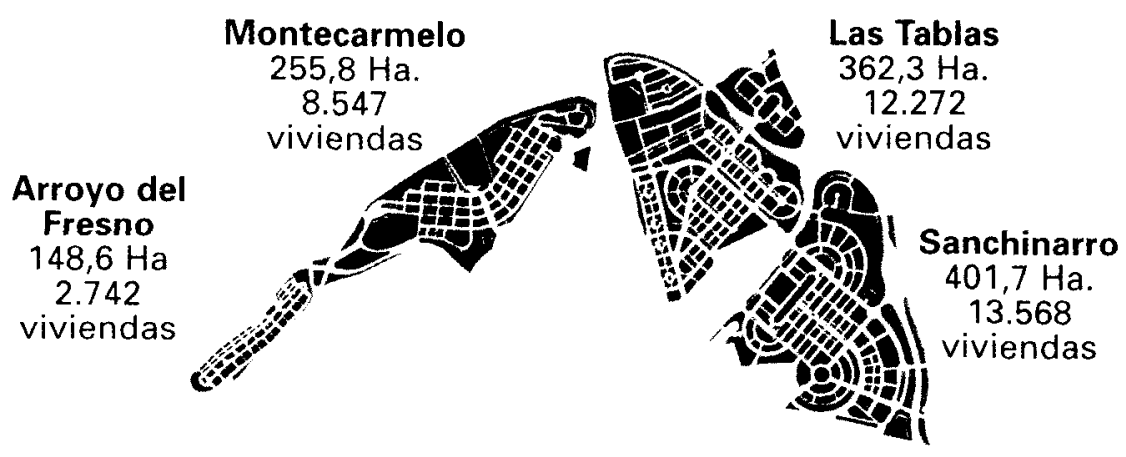

Fig. 2. Trama urbana y localizada de los PAUS del norte de la ciudad, respecto al continuo edificado. Fuente: Gerencia Municipal de Urbanismo (Ayuntamiento de Madrid, 1999a).

impacto medioambiental considerable sobre uno de los principales pulmones de la capital. El número de licencias concedidas era, en la fecha indicada, sensiblemente inferior; no alcanzando, en ningún caso, el $30 \%$ de las totales previstas.

El PAU de Arroyo del Fresno presenta un desarrollo lineal, paralelo a la $\mathrm{M}-40$, con una solución de ordenación que plantea la integración con el entorno del parque natural, en una graduación de densidades y alturas en disminución, dirigida desde el interior de la ciudad hacia las áreas del monte del Pardo. Es el que tiene una densidad de edificación más baja, en consonancia con la fragilidad del espacio natural que domina su borde norte. Su estructura urbana muestra el marcado contraste existente entre la zona más urbanizada, en el extremo norte, en la proximidad de la estación de Pitis, donde se sitúan los equipamientos comerciales y servicios terciarios del barrio, y el extremo sur, que se destinará a la creación de un campo de golf, que, a modo de barrera, intentará disminuir el impacto ambiental provocado por la urbanización.

EI PAU de Montecarmelo contiene un esquema urbano semejante al anterior, lo que no es de extrañar, habida cuenta de que no es sino su extensión natural, ocupando el último espacio existente entre el consolidado urbano actual y los parques naturales de El Pardo y de El Manzana- 
res. En el mismo, predomina la vivienda colectiva de cuatro plantas (88\%), completada por vivienda unifamiliar de dos plantas y vivienda aislada. En el barrio, domina el espacio residencial urbanizado (92\%) sobre el de servicios (8\%) (ASPRIMA, 2000a).

Finalmente, el PAU de Las Tablas, situado entre Sanchinarro y Montecarmelo, se desarrolla, básicamente, entre los polígonos industriales actualmente existentes. En el futuro, se construirá un paso superior sobre la autovía del norte (paso Renault) y dos más a distinto nivel permitirán salvar el trazado del ferrocarril hasta la avenida de los Infantes (fig. 3). La operación incluye el remate del polígono 18 de Alcobendas en la zona exterior a la $\mathrm{M}-40$.

Por su parte, los PAUs de Vallecas y Carabanchel contienen el valor añadido de aproximar la residencia a los grandes espacios industriales del este y del sur de la capital. EI PAU de Vallecas, también conocido como Ensanche de Vallecas, supone la operación urbanística de mayor calado de todas cuantas están, actualmente, en marcha. Sobre una superficie de 736 hectáreas, se construirá un volumen superior a las 26.000 viviendas,

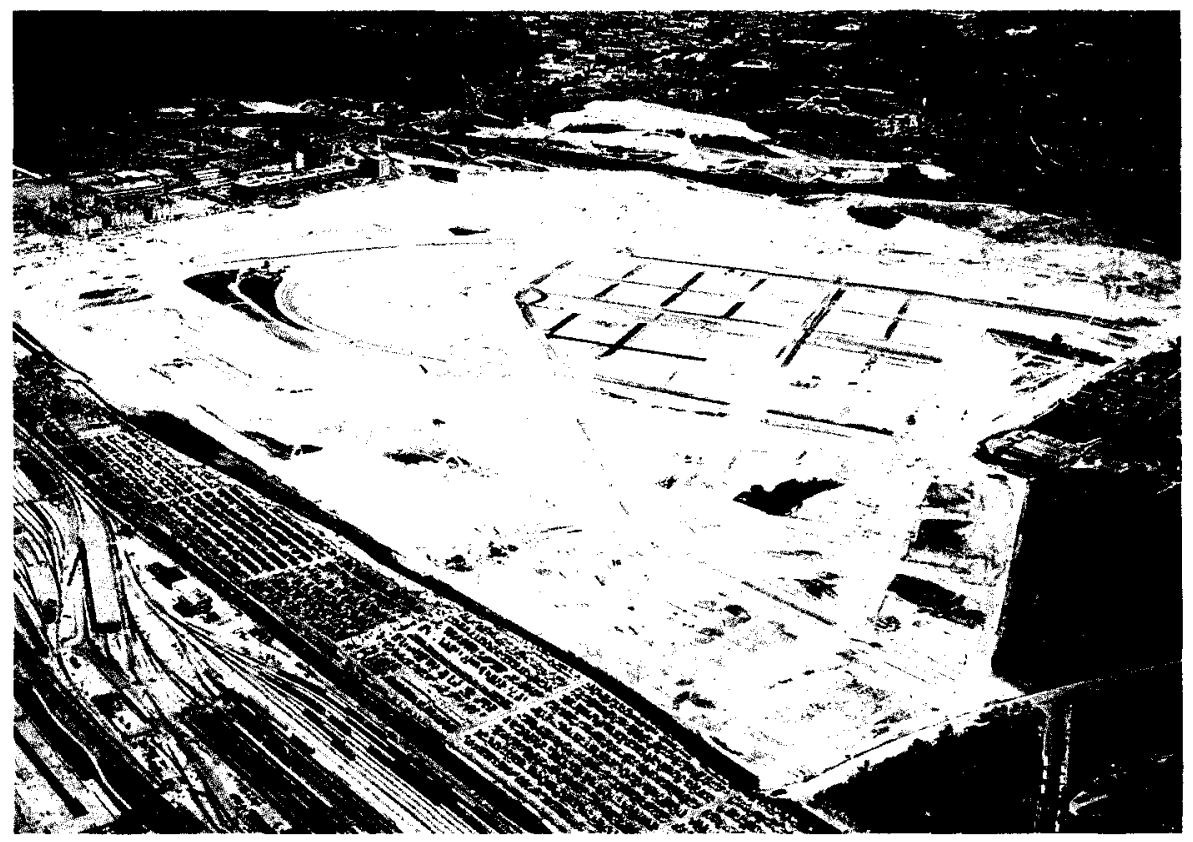

Fig. 3. Zona que ocupará el PAU de Las Tablas, en el borde de la M-40 y áreas industriales circundantes. Fuente: Gerencia Municipal de Urbanismo (Ayuntamiento de Madrid, 2002). 
la mayoría de tipología multifamiliar, lo que puede representar una de las más grandes reservas de suelo para vivienda protegida de la ciudad. Dentro de los equipamientos, destaca el proyecto de construcción de un gran centro comercial, sobre una extensión de 220 has.

Para concluir, en el extremo sur del popular barrio de Carabanchel, entre el límite de la ciudad edificada y el trazado de la autovía de la M-40, se halla el PAU de Carabanchel (fig. 4). Esta actuación urbanística supone el cierre del área urbanizada actual, resolviendo, de esta manera, los problemas de borde urbano de la zona. Al diseño del nuevo barrio se han incorporado dos nuevos enlaces viarios, la M-45, ya construida, y la R-5, en proyecto de construcción. La operación recuperará el uso colectivo del paraje histórico denominado "El Pinar de San José» y desarrollará, longitudinalmente, un gran parque lineal, en el borde de la $M-40$, que cumplirá la función de pantalla acústica (ASPRIMA, 2000b). El proyecto se halla dividido en tres Unidades de Ejecución, de las cuales se está edificando en la Unidad de Ejecución 2. En noviembre del pasado año, se habían concedido licencias para un total de 4.321 viviendas. Se espera que sea uno de los nuevos barrios que concluyan con mayor rapidez el desarrollo previsto.

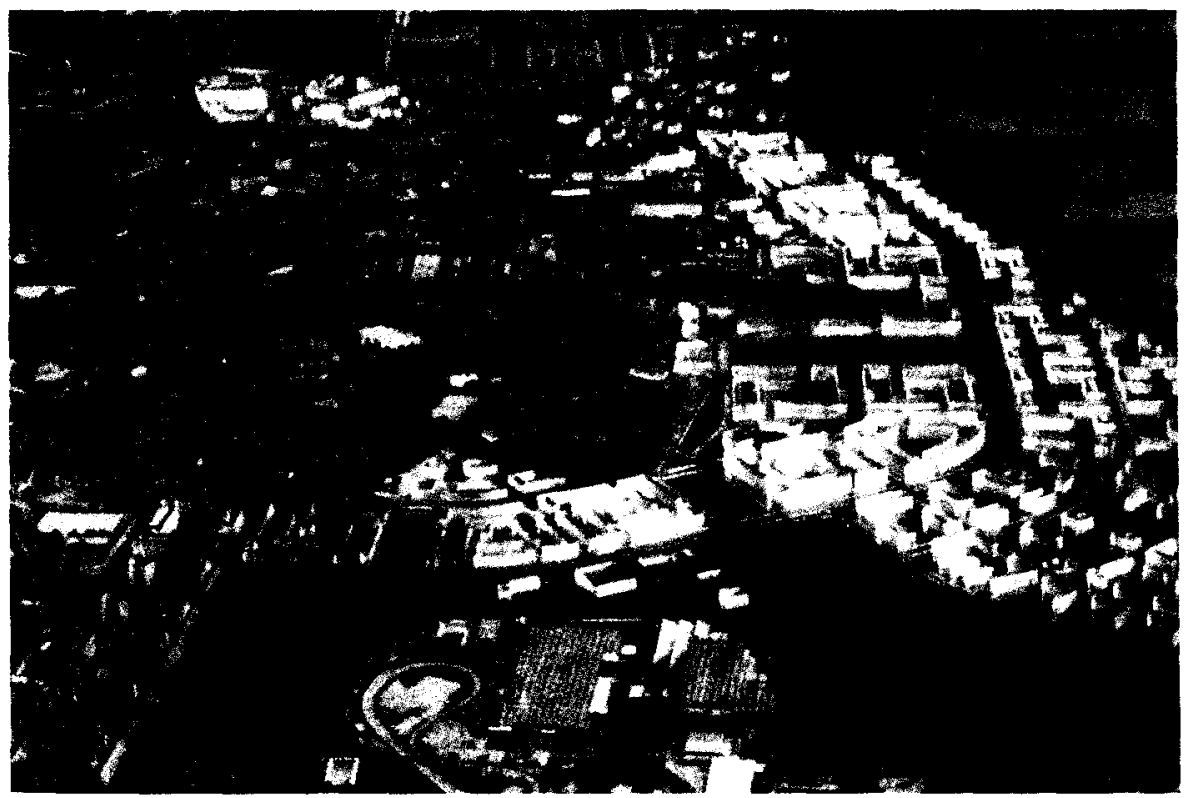

Fig. 4. Carabanchel, nuevo ensanche en el borde sur de Madrid (barrio en construcción). Fuente: Gerencia Municipal de Urbanismo (Ayuntamiento de Madrid, 2002). 


\section{UNA VALORACIÓN CRITICA DE LAS ACTUACIONES DESARROLLADAS POR LOS PAUS EN LA SOLUCIÓN DEL PROBLEMA DE LA VIVIENDA DE LA CAPITAL}

El estado actual de las operaciones urbanísticas, casi diez años después de iniciado el desarrollo de los PAUs, permite extraer una serie de importantes conclusiones sobre lo que para algunos autores no podía ser sino la historia de un fracaso anunciado ${ }^{5}$. Las razones son muchas y variadas y afectan a los múltiples aspectos que una política equilibrada de vivienda debe comprender.

Desde una perspectiva global, el primer gran fracaso ha sido, sin duda, el incremento del precio de la vivienda, que ha dejado de lado a un porcentaje cada vez mayor de la demanda no solvente, aquella que difícilmente puede acceder a la vivienda en propiedad si no es con ayudas gestionadas desde la administración pública. Los datos no pueden ser, a este respecto, más elocuentes. Durante los últimos años, la tendencia de crecimiento de los precios en las grandes aglomeraciones urbanas españolas, como Madrid o Barcelona, ha resultado ser exponencial (fig. 5). Este

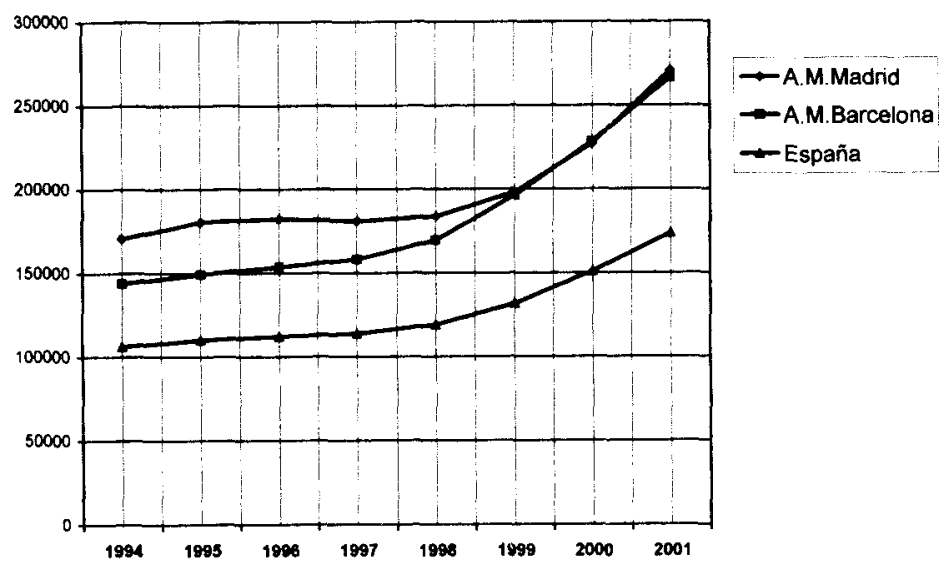

Fig. 5. Evolución de los precios de la vivienda (ptas./ $\mathrm{m}^{2}$ ) de las aglomeraciones urbanas de Madrid y Barcelona (1994-2001). Fuente: AGECOVI, "Informe sobre la coyuntura del mercado de la vivienda, año 2001".

5 Ya en una fecha tan temprana como 1996, E. MANGADA, antiguo consejero de la CAM por el grupo socialista, adelantaba gran parte de las negativas consecuencias que el planteamiento implícito de los PAUs tendría para la ciudad. 
hecho se ha producido, paralelamente, al continuado descenso de la producción de viviendas sociales en nuestro país, lo que ha agravado, aún en mayor proporción, la desprotección hacia los segmentos de la demanda más desfavorecidos (fig. 6).

La elevación del precio de la vivienda no ha sido sino la consecuencia de la política del suelo urbano del gobierno popular. La recalificación masiva de suelo en la capital madrileña ha dejado en manos privadas un jugoso negocio inmobiliario, lo que ha significaóo apostar por una manera determinada de construir la ciudad. En un primer momento, la expropiación constituyó el instrumento diseñado para la preparación y posterior gestión del suelo en las áreas ocupadas por los PAUs. La rectificación posterior y su sustitución por el procedimiento de compensación, en el que los propietarios de suelo promueven los nuevos barrios, ha permitido la acumulación de terreno en pocas manos y la especulación como forma de acumulación de capital. La importancia de esta mercancía, que constituye la clave para poder construir, así como su revalorización en los momentos de bonanza económica, son los factores que explican la estrategia de muchas promotoras inmobiliarias en la acumulación de suelo urbanizable y como la adquisición de suelo es otro negocio más, que sumar al de la promoción y venta de viviendas. Quien acapara suelo no lo vende hasta el momento en que los precios se estabilizan y la reducción del negocio aconseja su rotación en el mercado. De esta manera, el suelo se utiliza como moneda de cambio y para fines muy diferentes a los urbanísticos,

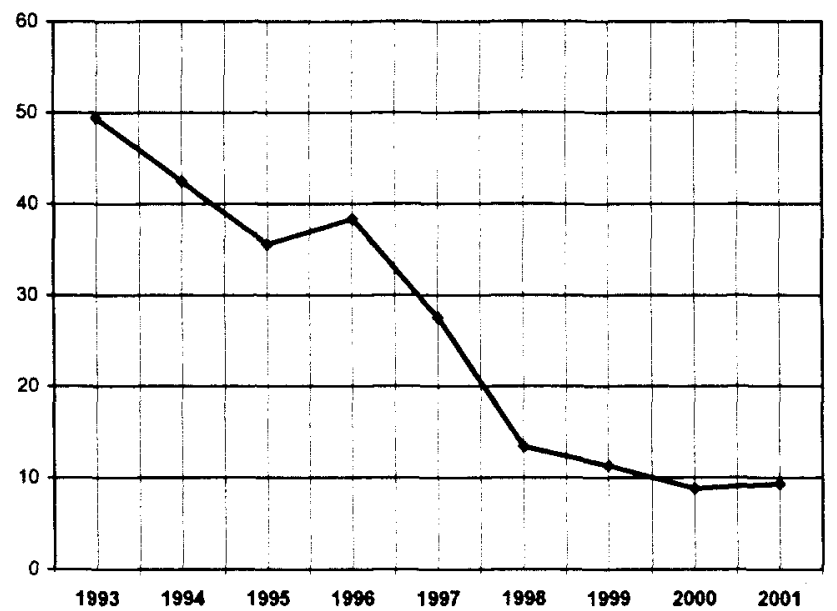

Fig. 6. Evolución del porcentaje de viviendas de protección oficial sobre el total de viviendas construidas en nuestro pais (1993-2001). Fuente: Ministerio de Fomento. 
empleándose, muchas veces, como pago a agentes que no son inmobiliarios, con lo que el efecto positivo de su potencial liberalización, en cantidad suficiente para la reducción de su precio, queda anulada.

Algunos datos, relativos al espacio residencial madrileño, ponen de manifiesto las tendencias apuntadas. Los precios del suelo en el mercado de rehabilitación de viviendas, en el centro de la capital, no bajan de los 2.700 euros por metro cuadrado, mientras que en áreas de desarrollo futuro, como los PAU del norte de Madrid, de Sanchinarro, Las Tablas o Montecarmelo, las parcelas de suelo para vivienda libre se venderían a unos 1.500 euros, con una repercusión en el precio de la vivienda que supondría la comercialización de un piso de tamaño medio por encima de los 270.000 euros ( 45 millones de pesetas de las de antes) ${ }^{6}$.

A esta desenfrenada carrera por la retención y especulación del suelo urbanizable se han sumado, en demasiadas ocasiones, las administraciones municipal y regional. La experiencia muestra, como en el territorio madrileño, parte de los ayuntamientos han participado, como un propietario más, en el proceso de buscar rentas en el proceso especulativo que se ha desatado durante los últimos años. Con esta finalidad, han utilizado la propiedad del suelo para la financiación de su presupuesto, abandonando la tarea de la formación de patrimonios de suelo municipal, desde donde relanzar una política de construcción de viviendas protegidas a precio reducido.

El resultado ha sido el encarecimiento exponencial del precio del suelo de la capital, en general, y de las nuevas áreas de construcción, en particular. Los datos reflejados en la fig. 7, relativos a la evolución de los precios del suelo urbano en los nuevos barrios, muestran como los mismos se ha multiplicado por siete, en las áreas del norte de la ciudad y casi por cinco en el este, según acreditan los registros de propiedad ${ }^{7}$.

Fracasada la política liberal de producción de vivienda, de "cuanto más suelo mejor, cualquiera que sea su localización, en la suposición de que la simple clasificación extensiva del suelo será suficiente para incrementar la oferta y abaratar los precios del suelo y la vivienda" (Mangada Simain, E., 1996), queda por valorar la política de protección oficial, fundamento esencial del primitivo proyecto de los PAUs. La falta de instrumentos de

\footnotetext{
6 Los datos empleados proceden de la información suministrada por la empresa Sociedad de Tasación S.A.

Los datos utilizados, sobre la evolución de precios del suelo urbano y su repercusión sobre el precio de la vivienda, vienen recogidos en el artículo: "El modelo de la vivienda del PP desata el fraude", publicado por el periódico «El País» el 4-11-2002.
} 


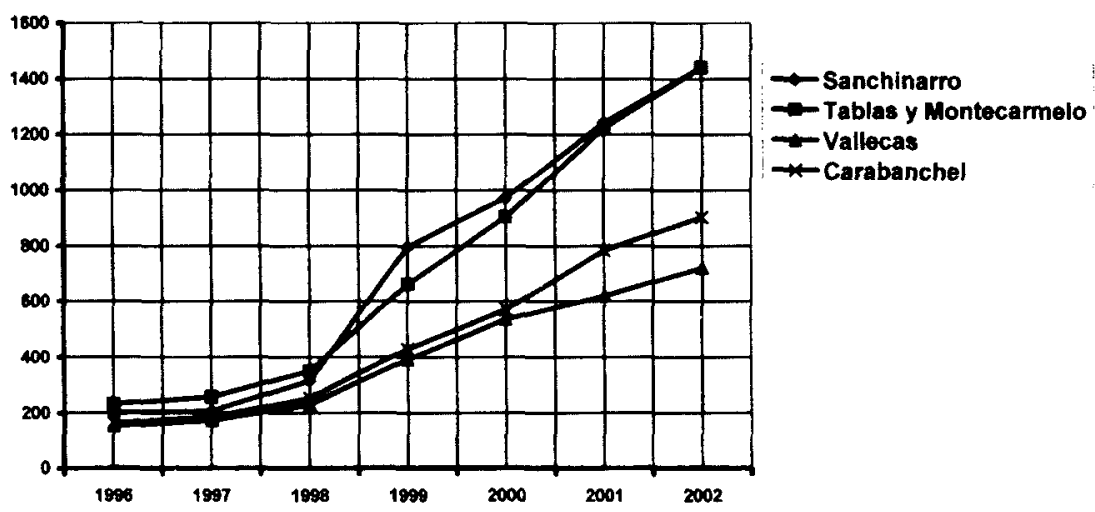

Fig. 7. Evolución del precio del suelo urbano (euros $/ \mathrm{m}^{2}$ ) en los barrios de nueva construcción de Madrid. Fuente: El País (4-11-2002).

control de los precios de la vivienda protegida y tasada, salvo por la limitación de los mismos en la escritura de compraventa, es la responsable de lo que todo el mundo conoce como un secreto a voces: que los precios son superiores a los valores escriturados, por el sobreprecio que se paga en dinero negro. Si bien es cierto que estas prácticas son difíciles de extirpar, por la aceptación que las mismas merecen en nuestra sociedad, una labor de inspección más exhaustiva podría tener efectos positivos que limitaran el fraude y permitieran que las adjudicaciones de viviendas fueran más justas y a un precio ciertamente controlado.

El resultado de la política aplicada al desarrollo de los PAUs de la capital no puede ser más desalentador. Los dos objetivos básicos que los propulsores del proyecto se plantearon no se han cumplido. En primer lugar, el abaratamiento de la vivienda en la capital. Los datos son suficientemente reveladores al respecto. Si bien es cierto que la confluencia de una serie de circunstancias, como la disminución de intereses de los créditos hipotecarios, la utilización de la vivienda como inversión, en un momento de descenso de rentabilidad de los fondos bancarios, el afloramiento de dinero negro, etc., han impulsado un mercado oligopólico como el del suelo y, por ende, el de la vivienda, en una condiciones de expansión de la demanda, a unas subidas de precios desorbitados, no es menos cierto que la política del "laser faire" del grupo popular ha favorecido la consecución de negocios inmobiliarios de manera descontrolada.

En segundo lugar, la puesta en el mercado, en un período de tiempo razonable, de viviendas protegidas dirigidas a las clases sociales más desfavorecidas, en el intento de reinvertir el éxodo de personas de la capital 
hacia la periferia. La acumulación de terrenos, en pocas manos, ha conseguido agrandar enormemente los plazos previstos, al regular la salida al mercado de las viviendas al ritmo deseado por los promotores de este tipo de residencia.

Los nuevos datos del Censo de 2001, para la capital y municipios aledaños, muestran como gran parte de los rasgos del modelo territorial madrileño, gestado en etapas anteriores, continúa manteniéndose. Si bien es cierto que el crecimiento de la capital ha resultado positivo, durante el último lustro, desde 1996, una parte importante del mismo se ha debido al incremento de la población inmigrante extranjera, que habita en Madrid en unas condiciones infrahumanas, en viviendas sobreocupadas, por las que pagan alquileres exagerados. Así mismo, la construcción de más de 100.000 viviendas en los años transcurridos desde la aprobación del Plan General, ha servido para consolidar barrios como Valdebernardo (Vicálvaro), Las Rosas (San Blas) o Madrid Sur (Vallecas), donde se ha contenido a una parte importante de la población de menor nivel de renta, aunque de manera insuficiente para dar respuesta a una demanda insatisfecha, que ha ido creciendo de forma desmedida durante los últimos años. Sin embargo, continúan siendo los municipios de la periferia madrileña, los elegidos por la demanda de menor nivel de renta como lugares de asentamiento. En este sentido, destacan los núcleos urbanos del corredor del Henares (Alcalá de Henares y Torrejón de Ardoz), así como los de mayor tamaño del arco sur de la periferia (Fuenlabrada, Parla y Pinto), con índices de crecimiento superiores al $10 \%$, donde se han concentrado el mayor contingente de la población migratoria procedente del centro de la ciudad.

\section{REFERENCIAS BIBLIOGRÁFICAS}

ASPRIMA (2000a): "PAU de Monte Carmelo", en Revista de la Asociación de Promotores Inmobiliarios de Madrid. $\mathrm{N}^{\circ}$ 10, julio, págs. 14-17, Madrid.

ASPRIMA (2000b): "Ensanche de Carabanchel", en Revista de la Asociación de Promotores Inmobiliarios de Madrid. N. 12, diciembre, págs. 15-20, Madrid.

AYUNTAMIENTO DE MADRID (1999a): Cuatro años de gestión urbanística 1995-1999. Gerencia Municipal de Urbanismo, Madrid.

- (1999b): La transformación de la ciudad en veinte años de ayuntamientos democráticos. Gerencia Municipal de Urbanismo, Madrid.

- (2002): Cuatro años de gestión del Plan de Ordenación Urbana de Madrid de 1997. Gerencia Municipal de Urbanismo, Madrid.

COMUNIDAD DE MADRID (1995): La economia de Madrid. Análisis espacial de las actividades económicas de la región. Consejería de Política Territorial, CAM. Madrid.

- (1998): Plan Regional de Estrategia Territorial. Plan Sectorial de la Vivienda. Consejeria de Obras Públicas, Urbanismo y Transporte, CAM, Madrid.

GRUPO MUNICIPAL SOCIALISTA (1993): El crecimiento de Madrid: una decisión insensata. Oficina Municipal del Plan. Gerencia Municipal de Urbanismo. Madrid. 
- (1996): Operación PAUs: modificaciones de PAUs y Planes Parciales de los ámbitos de Montecarmelo, Las Tablas, Sanchinarro, Carabanchel, etc. Oficina Municipal del Plan. Gerencia Municipal de Urbanismo. Madrid.

MANGADA Simain, E. (1996): “ ¿Suelo para Madrid? Jornadas del Nuevo Plan General. Gerencia Municipal de Urbanismo. Madrid

Martín Valdivia, S. M. (1998): Urbanismo y especulación. Los patrimonios públicos de suelo. Editorial Montecorvo, Madrid.

MINISTERIO DE OBRAS PÚBLICAS Y TRANSPORTES Y MEDIO AMBIENTE (1996): Informe sobre suelo y urbanismo en España. MOPTMA, Madrid.

NASARRE GoICOECHEA, F. (1995): «PAUs: Programas de Actuación Urbanística para afrontar el problema de la vivienda en el municipio de Madrid" en Urbanismo. Colegio Oficial de Arquitectos, págs. 6-25, Madrid.

Ortega Garcia, A. y Ortega Cirugeda, J. (2001): Comentarios a la Ley del Suelo de Madrid. Editorial Montecorvo, Madrid.

SANTOS PRECIADO, J.M. (2001): "Las periferias urbanas y la organización de la ciudad actual: el caso de Madrid" en Ciudad y Territorio. Estudios Territoriales. Ed. MOPU, Madrid, págs. 669-688. 